\title{
Los sindicatos ante la conciliación de la vida laboral y familiar-personal
}

\author{
Fausto Miguélez \\ Josep Maria Antentas \\ Oriol Barranco \\ Dafne Muntanyola
}

Universitat Autònoma de Barcelona. Departament de Sociologia

Grup d'Estudis Sociològics sobre la Vida Quotidiana i el Treball

08193 Bellaterra (Barcelona). Spain

Fausto.Miguelez@uab.cat

Josepmaria.Antentas@uab.cat

Oriol.Barranco@uab.cat

Dafne.Muntanyola@uab.cat

\section{Resumen}

En este artículo se analizan las posturas de los sindicatos ante la conciliación, a partir, tanto de documentos oficiales legislativos europeos y españoles, como de entrevistas realizadas a afiliados sindicales. Así, vemos como la postura sindical, la articulación de la conciliación como un derecho colectivo no conlleva una conciliación real entre la vida laboral, la familiar y la personal, dada su identificación como medida para mujeres trabajadoras, sin implicar una nueva organización del trabajo, tanto productivo como familiar, ni tampoco un aumento de servicios públicos asistenciales.

Palabras clave: conciliación, negociación colectiva, derecho colectivo, individualización, políticas de igualdad y de género.

\section{Abstract. Work-life balance: the unions' position}

Through both the exploitation of European and Spanish official documents, jointly with interview analysis carried out among Spanish labor unionists, the position of labor unions around work-life balance policies is explained. The analysis achieved in this article puts forward that the formulation of work-life balance as a collective right does not seem to imply a real balance among the productive, the domestic and the personal dimensions of working life. In all, its identification as a women-only policy does not promote a new organization of work, domestic as well as productive, or an increase of public aid services.

Key words: work-life balance, collective bargaining, collective right, individualization, equality and gender policies.

\section{Sumario}

\section{Introducción}

2. El sindicalismo europeo y las políticas de conciliación de la vida laboral y la vida familiar

3. Los sindicatos españoles ante la conciliación
4. Fuerza y límites de la actuación de los sindicalistas sobre esta cuestión

5. A modo de conclusión

Bibliografía 


\section{Introducción}

La cuestión de fondo, cuando hablamos de conciliación entre la vida laboral, familiar y personal, es la relación entre los tiempos de la vida y el bienestar que de su uso se debería derivar. Hay un tiempo del día o de la semana que la mayoría de las personas tiene que dedicar al trabajo remunerado, porque de ahí obtiene los recursos necesarios para vivir. Según la perspectiva histórica, ese tiempo se ha ido reduciendo, aunque diferenciadamente según grupos sociales, desde el principio de la revolución industrial —3.000 horas al añohasta hoy -1.700 como media en la Unión Europea-. Hay un tiempo de «trabajo necesario» (para el cuidado de uno mismo y de los demás) que no es tan claro que lleve la misma dirección de reducción, puesto que si las tecnologías permiten acortar ciertas actividades (lavar, cocinar, limpiar), las que implican cuidado de las personas requieren más tiempo, es decir, tenemos menos hijos pero su educación supone más dedicación, la esperanza de vida se alarga y, por tanto, son más los ancianos o los discapacitados que requieren cuidados. Hay un tercer tiempo, el de «libre disposición», que se ha visto incrementado, pero que deseamos tener cada vez en mayor cantidad, porque muchas de las actividades que nos gustaría hacer lo que requieren, aparte de recursos, es tiempo.

Se da una tensión entre los tres tiempos que tradicionalmente ha sido «resuelta» por la sobrededicación de un grupo de personas, las mujeres que son amas de casa. Pero resulta que esas personas quieren estar cada día más presentes en el mercado de trabajo y desean también disfrutar del tercer tiempo, porque es su derecho, aparte de tener sus estatus de madres, abuelas o esposas. He ahí el conflicto que ha desencadenado la necesidad y la exigencia de la conciliación. La Unión ha querido hacerle frente con la línea de «igualdad de oportunidades» reflejada en las políticas de empleo que se desarrollan desde el Tratado de Amsterdam. Probablemente, el razonamiento de fondo de quienes han ideado estas políticas ha sido que las mujeres quieren estar cada día más presentes en el mercado de trabajo, lo que las aleja de su rol tradicional y las induce a tener menos hijos, lo cual está provocando el envejecimiento de la sociedad europea. Por tanto, un aspecto importante de la «igualdad de oportunidades» que se propone, consiste en que las mujeres puedan conciliar su tiempo de trabajo remunerado con su tiempo familiar, lo que incentivaría la natalidad. Es decir, las políticas oficiales de conciliación están pensadas principalmente para las mujeres madres. Las legislaciones de conciliación despliegan una serie de posibilidades en ese sentido: permisos y subsidios de maternidad, horas para el cuidado de niños, entrada y salida flexible al trabajo, guarderías de empresa, pero diferentes actores de la sociedad civil piensan que ese derecho de conciliación debe alcanzar a todas las mujeres y a todos los hombres, porque ése sí sería un paso muy importante en la igualdad de oportunidades.

Las hipótesis de las que partimos pueden formularse de esta manera: a pesar de que las políticas de conciliación en la UE nacen con el apoyo de los sindi- 
catos, éstos siguen siendo críticos con un concepto muy restringido de las mismas que las referiría principalmente a las mujeres trabajadoras. En la prácti$\mathrm{ca}$, sin embargo, los sindicalistas se acercan mucho más a este punto de vista que hoy día es el mayoritario de los propios trabajadores y trabajadoras ${ }^{1}$.

\section{El sindicalismo europeo y las políticas de conciliación de la vida laboral y la vida familiar}

El sindicalismo europeo ha tenido y tiene una visión favorable de las políticas de conciliación entre la vida laboral y la vida familiar, promovidas desde las instancias europeas ya en los años noventa. Estas políticas emanan de directivas y medidas impulsadas desde la UE, en particular de las directivas 92/85/EC del 19 de octubre de 1992, sobre maternidad, y 96/34/EC del 3 de junio de 1996 , sobre permisos parentales. Ésta última es fruto de un acuerdo previo entre la Confederación Europea de Sindicatos (CES) y las patronales europeas, UNICE y CEEP, del 14 de diciembre de $1995^{2}$.

Las directivas comunitarias han sido la base para las posteriores políticas y adaptaciones y cambios legales en aquellos países de la UE que carecían de políticas al respecto o cuyas políticas vigentes no se adaptaban al nuevo marco. Ejemplos de estas trasposiciones legales al ámbito nacional de las directivas europeas los encontramos, para citar algunos, en el caso de la Ley sobre conciliación entre la vida laboral y la vida familiar (1999) en el Estado español, la legislación sobre permisos parentales aprobada en Gran Bretaña en 1999, la Ley sobre permisos parentales (Legge di Congedi Parentali) en Italia (marzo de 2000), o su homóloga en Alemania (julio de 2000) . $^{3}$ La adaptación en cada país se ha hecho mediante cambios legales y nuevas disposiciones consensuadas entre los actores sociales y con el acuerdo, por tanto, de los sindicatos, que han mantenido una orientación globalmente favorable hacia las mismas. En el caso británico, la legislación sobre permisos parentales de 1999 contó con el apoyo de la TUC, considerando las medidas aprobadas como un primer paso positivo pero insuficiente (Math y Meilland, 2004). En el caso italiano, los sindicatos consideraron la ley como un gran paso adelante y una conquista, mientras que la Confindustria, la organización patronal, rechazó su contenido (Trentini, 2000). En el caso alemán, la ley fue aprobada con algunas críticas por parte de la BDA, la organización patronal alemana, y con el apoyo de la DGB, si bien ésta señaló algunas carencias de la misma, como el hecho que

1. Para redactar este artículo, hemos recurrido a los documentos oficiales y oficiosos de los sindicatos, tanto europeos como españoles, así como a entrevistas a sindicalistas en empresas y sectores (véase la nota introductoria de este número monográfico en la que se explica la metodología de la investigación).

2. Ver: «Framework agreement on parental leave», UNICE, CEEP y CES, 14 de diciembre de 1995.

3. Para un a visión más detallada y exhaustiva de las diferentes medidas y cambios legales adoptados en los países de la UE, ver: Rossi y Demetriades (2002). 
algunas de las medidas que la Ley contemplaba no eran aplicables en empresas menores de dieciséis trabajadores (Schulten, 2000).

Las políticas de conciliación nacen, por tanto, con el apoyo del sindicalismo europeo y la CES, y de las organizaciones sindicales nacionales en sus adaptaciones nacionales. Conviene recordar, sin embargo, que en la aplicación de las directivas europeas en cada país, existen distintos acentos y matices. En los países nórdicos, por ejemplo, se ha puesto más acento en la necesidad que los hombres se impliquen más en las tareas familiares y domésticas, mientras que en la mayoría de los otros países europeos se parte en general, de forma más o menos implícita, de la idea que son las mujeres quienes deben «arreglárselas» para combinar los ámbitos laborales y domésticos (Heinen, 2004). Como veremos posteriormente, esta "duplicidad» se puede detectar también en los sindicatos españoles. Responde a una cultura dominante de predominio machista en la división del trabajo que no sólo está enraizada entre los hombres, sino también asumida pasivamente por muchas mujeres.

$\mathrm{El}$ análisis del posicionamiento del sindicalismo europeo en materia de políticas europeas de conciliación debe enmarcarse, para ser más comprehensivo, en un análisis más general de las posiciones sindicales frente a las políticas europeas de empleo. De forma sintética, la posición de la CES y del grueso del sindicalismo europeo en materia de política de empleo, y también en la cuestión más concreta de las políticas de conciliación, puede resumirse en un «acompañamiento crítico» de las políticas en marcha. Es decir, de apoyo a las mismas, de participación activa a menudo en el diseño de sus contenidos y, al mismo tiempo, de considerar que su contenido es insuficiente o su aplicación no del todo satisfactoria. El apoyo del sindicalismo europeo a las políticas de empleo de la UE es fruto de la estrecha relación existente entre el propio desarrollo del sindicalismo europeo y de sus estructuras, y el desarrollo del proyecto de integración europea y de la UE, en particular desde mediados de los ochenta, con la promoción del «diálogo social» desde la Comisión Europea, y el reconocimiento de la CES como interlocutor europeo, como han analizado diversos autores como Gobin (1997) o Martin y Ross (2001).

La CES, y las organizaciones nacionales que la conforman, ha valorado positivamente y apoyado la Estrategia Europea de Empleo establecida en la Cumbre de Luxemburgo en diciembre de 1997, así como después la Estrategia de Lisboa, establecida en el Consejo Europeo de Lisboa en marzo del año 2000, cuyo objetivo nominal declarado es convertir la UE en la economía más dinámica y competitiva del mundo, capaz de abordar un crecimiento sostenible, con más y mejor empleo y mejor cohesión social en el horizonte del 2010. En paralelo al apoyo general a la Estrategia de Lisboa, la CES ha manifestado en distintas ocasiones su preocupación por una aplicación «unilateral» y «sesgada» de la misma, centrada primordialmente en la desregulación y flexibilización del mercado laboral y la liberalización de los mercados (CES, 2004a y 2004b). Conviene hacer notar que esta posición contrasta con la de varios autores (Etxezarreta, 2001), que han señalado cómo las políticas de flexibilización y desregulación forman parte integral del contenido de la Estrategia de 
Lisboa, cuyo objetivo de creación de ocupación y de alcance del "pleno empleo» se basa en la creación de trabajos de «mala calidad» y en la reducción de la prestaciones sociales. Para ser más precisos, quizá se ha tratado de crear empleo sin tener en cuenta ciertas características del mismo que tienen que ver con la estabilidad y la seguridad. Algunas publicaciones de la Comisión (Commission Européenne, 2002) dan una gran importancia a la «satisfacción» y a la «voluntariedad» en la calificación de un empleo como bueno, olvidando que la gente puede mostrarse satisfecha con un empleo precario, porque la alternativa es no tener ninguno.

Este enfoque de fondo sobre la política de empleo de la UE también va en correspondencia con el enfoque concreto que la CES y el grueso del movimiento sindical europeo mantiene específicamente en materia de política de conciliación. En este capítulo, la CES considera que la directiva sobre permisos parentales y las medidas que se han derivado de la misma en los países de la UE son positivas y suponen pasos adelante en la cuestión de la conciliación entre vida laboral y familiar. Sin embargo, señala algunas insuficiencias importantes de las políticas aplicadas en este terreno. En opinión de la CES, los permisos son solamente uno de los aspectos de las políticas de conciliación, y éstos deben ir acompañados de medidas más generales, como una política de promoción de servicios sociales de cuidado de niños y ancianos que, hoy en día, no se dan de forma suficiente ni adecuada en los países de la UE. Estas políticas, tal y como se establece en el Plan de Igualdad de Género y en el Programa de Acción aprobado en el último congreso de la CES (Praga, mayo del 2003), deberían trascender su actual «etiqueta» de medidas sobre conciliación de la vida laboral y familiar para pasar a ser parte integral de las políticas de reforma del Estado de bienestar. En definitiva, deberían salir de la estricta «esfera de género" para ser integradas en un conjunto de políticas más generales (Tiernan, 2004). La CES misma queda prisionera de una concepción que va en la línea de «mejorar la situación de las mujeres», insuficiente porque en el fondo asume la división sexual del trabajo existente. Algunos autores, desde un enfoque más crítico con los límites de las políticas de conciliación que el de las organizaciones sindicales, recuerdan que las políticas de conciliación nacen en un contexto, el de los años noventa, marcado por la hegemonía de las políticas neoliberales que insisten en la flexibilización del mercado de trabajo y que otorgan mucha centralidad a la cuestión de la individualización de las responsabilidades dentro de la esfera privada (Heinen, 2004). Al mismo tiempo, también han señalado ampliamente que las tendencias actuales en el mercado de trabajo y en las políticas de empleo (desregulación horaria, flexibilización, creación de empleo de «mala calidad»...) van en sentido contrario a las necesidades de las personas, y de las mujeres, de conciliar la vida personal y la laboral (Rubery, 1997).

La CES considera positiva la estrategia de gender mainstreaming impulsada por la UE en materia de políticas de igualdad entre hombre y mujer, e incorporada en 1999 en la Estrategia Europea de Empleo. Ésta enmarca las políticas de género en un enfoque transversal más general, más allá de las polí- 
ticas de género específicas, de forma que las políticas de igualdad, aparte de ser objeto de medidas específicas en el ámbito del cuarto pilar de las políticas de empleo, el de «igualdad de oportunidades», también deber ser contemplada en un enfoque integral y transversal en los otros tres pilares, empleabilidad, enterpreneurship, y adaptabilidad. Algunas autoras más críticas, sin embargo, como Hubert (1998) o Heinen (2004), han señalado que este enfoque más "global» de las políticas de género puede tener algunos efectos nocivos, como acabar «diluyendo» el problema, y que sus beneficios sólo se den en aquellos países con una fuerte política previa en el terreno de la igualdad entre hombre y mujer.

Desde nuestro punto de vista, se necesitan ambas cosas al mismo tiempo: políticas de igualdad y políticas de género, las primeras para poder hablar con propiedad de personas con los mismos derechos y las mismas obligaciones, las segundas para colmar lo más rápidamente posible la gran distancia que hay entre las oportunidades de los hombres y las de las mujeres.

\section{Los sindicatos españoles ante la conciliación}

La investigación a la que se refiere este artículo quiere captar la política de los sindicatos en las empresas respecto a la conciliación. Para ello, conviene también examinar los documentos oficiales de las dos centrales sindicales mayoritarias en el Estado español: CCOO y UGT.

\section{En los documentos sindicales oficiales ${ }^{4}$}

En los documentos sindicales oficiales analizados, el término conciliación aparece primero en los documentos congresuales de ambas centrales sindicales,

4. El posicionamiento de los sindicatos se ha extraído de la revisión de los documentos que se citan a continuación.

En lo que respecta a CCOO: Balance de la Negociación Colectiva 2001, Secretaría Confederal de Acción Sindical CCOO, abril de 2002; Criterios para la Negociación Colectiva para 2003, Comisión Ejecutiva Confederal CCOO, 2002; La conciliación de la vida laboral y familiar en los convenios colectivos, Secretaria Confederal de la Mujer CCOO; Informe General, Programa de Acción y Estatutos del VIII Congreso Confederal CCOO, Madrid, diciembre de 2003; Informe General, Estatuts i Propostes d'Acció del VII Congrés de la Confederació Sindical de la CONC, abril de 2004; Resolución del II Congreso de FITEQA, CCOO sobre el Plan de Igualdad, noviembre de 2000.

Por lo que se refiere a UGT, los documentos revisados han sido: el Programa de acción de UGT confederal del $38^{\circ}$ Congreso Confederal de UGT de marzo de 2000; las Resolucions de l'Últim Congrés d'UGT-Catalunya, 2000, y las Resoluciones del Congreso de FIA, UGT, 2000. Además, se han revisado los dos siguientes documentos comunes de ambas centrales sindicales: Objetivos prioritarios para la negociación colectiva y la renovación del ANC 2005 (CCOO-UGT), y Consideraciones generales y buenas prácticas sobre la igualdad de oportunidades entre hombres y mujeres en la negociación colectiva. Aprobado por la comisión de seguimiento del acuerdo sobre Negociación Colectiva, febrero de 2003; Propuestas de CCOO y UGT para la negociación con CEOE y CEPYME sobre los criterios para la Negociación Colectiva en 2003 (ANC), diciembre de 2002. 
tanto a nivel estatal como territorial o autonómico. En el 2005, aparece en el marco de los objetivos para la negociación colectiva, donde se reconoce la necesidad de intervenir en la organización del trabajo para conciliar la vida laboral y familiar, mencionando especialmente las dificultades del colectivo femenino. El hecho de que la terminología utilice habitualmente el término familiar introduce una cierta desviación de entrada.

A partir del análisis realizado de dichos documentos, mostramos a continuación, en primer lugar, la importancia que tiene la cuestión para los sindicatos, así como una determinada concepción de lo que, en general, se considera que es y lo que significa la conciliación. En segundo lugar, se señalan cuáles son las medidas concretas que proponen para facilitar dicha conciliación. Finalmente, en tercer lugar, se definen las estrategias y los frentes de acción para conseguir que se establezcan medidas para la conciliación.

\section{Aparición del concepto en los documentos sindicales}

La conciliación aparece como un objetivo a conseguir en la negociación colectiva, pero siempre en un segundo plano respecto a los objetivos relacionados con la creación y el mantenimiento de empleo, así como a las cuestiones relativas a las condiciones de trabajo. Este orden de prioridades queda reflejado en los documentos sindicales de ambos sindicatos, así como en el documento común de Objetivos prioritarios para la negociación colectiva del 2005.

Para ambas centrales sindicales, la organización del trabajo y la organización del tiempo que de ella deriva son cuestiones centrales que deben ser abordadas para poder enfrentarse a la necesidad de la conciliación. La reducción y reorganización del tiempo de trabajo se consideran centrales, en primer lugar, para fomentar el empleo, pero, en segundo lugar, también para favorecer la conciliación. Para ello, ambos sindicatos defienden la necesidad de intentar conseguir tener una mayor participación y control en las decisiones empresariales sobre la organización del trabajo.

En los documentos, aparece una cierta tensión entre las distintas concepciones de la conciliación. Si, en los dos últimos años, el término aparece como una forma de organización laboral, con especial atención al tiempo de trabajo, también figura junto a otras medidas de igualdad de oportunidades, normalmente bajo capítulos específicos para las mujeres trabajadoras, que priorizan los mecanismos de no-discriminación en el puesto de trabajo que pueden fácilmente entenderse - y así lo entienden con frecuencia sindicalistas y trabajadores - como medidas de apoyo a la integración laboral femenina. Además, la demanda de una mayor regulación y de un incremento de bienes y servicios asistenciales identifica muchas de las reivindicaciones asociadas a la conciliación con medidas externas al mercado laboral. Se podría especular sobre los posibles efectos perversos de esta tensión conceptual, que, al no cuestionar realmente el rol social de las trabajadoras en la división sexual del trabajo, mantiene a éstas en una posición subordinada al colectivo de trabajadores. 


\section{Medidas concretas}

Dado que los procesos de flexibilidad laboral son vistos como uno de los elementos centrales de la negociación colectiva para intentar conseguir la plena ocupación, los documentos de ambos sindicatos también piden el establecimiento de criterios de preferencia para las mujeres en los casos en que el nivel de temporalidad les afecte más que a los hombres. Es decir, se toma conciencia de que numerosas situaciones de debilidad o de dependencia familiar, como las familias monoparentales, tienen a las mujeres como único sustento. Además, también se plantea el tiempo parcial como una opción, y no como una obligación, para que el conjunto de oportunidades de las mujeres no sea más restringido que el de los hombres. La opcionalidad o voluntariedad debe ser contrastada con rigor, porque, con frecuencia, las normas dominantes hacen que las mujeres entiendan que primero está la obligación, que es el trabajo doméstico, y que después puede haber un trabajo "voluntario» a tiempo parcial. De hecho, se considera necesario el control de la flexibilidad horaria para evitar prolongaciones injustificadas y distribuciones irregulares, que discurran bajo criterios arbitrarios nocivos para la organización personal y familiar de los trabajadores y de las trabajadoras. El caso más evidente lo tenemos en el comercio, donde las horas extraordinarias, para ordenar y cerrar, pueden alargarse a voluntad de la dirección o del dueño. En conjunto, esa doble cara de la flexibilidad laboral, de contrato y horaria, es un elemento considerado central para asegurar la igualdad de oportunidades entre ambos sexos.

Los capítulos dedicados a los permisos parentales y de maternidad ocupan el primer puesto en todas las secciones dedicadas a la igualdad de oportunidades entre mujeres y hombres, independientemente de la inclusión o no del término conciliación. En conjunto, se perciben como un sistema de regulación temporal, asociándose con las reivindicaciones prioritarias entorno a la flexibilidad laboral. Dentro de las medidas a adoptar, se sugiere la contratación de interinos y la individualización de los permisos de parentalidad, para superar así una habitual neutralidad que lleva, en la práctica, al no aprovechamiento de los permisos por parte de los padres, sino sólo por parte de las madres.

\section{Estrategias y frentes de acción}

El discurso de las centrales sindicales plantea conseguir unas condiciones laborales que permitan la conciliación de los trabajadores y las trabajadoras mediante la acción por tres vías:

1) En la negociación colectiva, mediante la introducción, en los convenios colectivos, de los derechos reconocidos por ley y, en los casos en los que sea posible, mediante la introducción en los mismos de medidas que mejoren la legislación vigente respecto a la conciliación.

2) A través de la presión política, destinada a conseguir que la legislación laboral reconozca en los derechos laborales un marco que facilite la conciliación. En este sentido, se debe destacar que ambos sindicatos consideran la 
Ley de conciliación vigente desde el año 1999 como insuficiente. La negociación colectiva puede ser más eficaz, porque da una agilidad que la ley no suele tener (Dikens, 2000).

3) Finalmente, ambas centrales sindicales consideran necesario luchar para conseguir que las administraciones públicas ofrezcan más y mejores servicios sociales que faciliten que las personas puedan conciliar su vida laboral con la familiar y/o personal. En este sentido, se señalan como centrales los servicios de guarderías y la mejora de los transportes públicos; la mejora de éstos últimos se considera necesaria para facilitar el acceso de los trabajadores y las trabajadoras a todos los polígonos industriales y el transporte hacia el puesto de trabajo a los trabajadores y a las trabajadoras de servicios o turnos nocturnos. Respecto a las guarderías, es importante subrayar el posicionamiento diferenciado que mantienen CCOO y UGT acerca de las denominadas guarderías de empresa o de los centros de trabajo. Mientras que CCOO se muestra contraria a esta medida por los posibles efectos perversos que se le pueden asociar, UGT considera que es una medida que puede permitir complementar la insuficiencia de la oferta pública existente y ser, por ello, útil.

\section{El nivel sectorial}

De forma general, no parece que se haya desarrollado ni que se esté desarrollando, en ninguna de las dos centrales sindicales, un discurso diferenciado a nivel sectorial sobre la cuestión que nos ocupa. Así, en los casos en los que existen documentos propios de las federaciones, no hay un trabajo de elaboración de discurso sindical propio de las federaciones: se asumen los planteamientos que, a nivel catalán y estatal, se aprueban sin realizar un trabajo de contextualización para el sector.

Sin embargo, de forma más minoritaria, podemos hablar de propuestas propias a nivel sectorial, como es el caso de los documentos de CCOO para el sector de químicas. Asumiendo la alta masculinización del sector, se define el proceso de transformación que se está dando con la incorporación de mujeres y se proponen medidas compensatorias e igualitarias para conciliar la vida laboral y familiar. Si bien son medidas que hacen referencia principalmente a la actividad sindical, existe la conciencia de la necesidad de una política transversal de género específica para el sector.

\section{Fuerza y límites de la actuación de los sindicalistas sobre esta cuestión}

Vamos a analizar ahora las opiniones y la actuación de los sindicalistas en las empresas, es decir, allí donde la conciliación puede provocar opciones diferenciadas de las empresas y de los trabajadores, pero también dentro de este último colectivo. No siempre resulta fácil diferenciar «ideas» $\mathrm{y}$ "prácticas» en esta cuestión, puesto que las primeras pueden ser frecuentemente meras legitimaciones de las segundas. 


\section{Lo que entienden los sindicalistas en la empresa por conciliación}

Lo que entienden los actores sociales en la empresa por «conciliación de la vida laboral y familiar» (en este caso nos basamos en una serie de entrevistas), es fundamental para comprender las prácticas y las estrategias en torno a esta cuestión. La directiva europea de conciliación nace como uno de los aspectos de las políticas de igualdad en el trabajo entre hombres y mujeres, como un mecanismo para ayudar a éstas últimas a superar el handicap de tener que cargar con los "deberes domésticos» al tiempo que desarrollan iniciativas para entrar o mantenerse en el mercado de trabajo (Lozares, 2004; Rigby, 2004; Torns, 2004). Esta directiva debe enmarcarse, como hemos señalado anteriormente, en el contexto de una estrategia europea de políticas de empleo que no tiene en cuenta ciertas características del mismo, como la estabilidad y la seguridad. En nuestro análisis, partimos de la concepción de los sindicatos, pero ésta se construye con referencia a las de los otros sujetos presentes: empresarios, trabajadores y Administración. En los sindicatos, podemos diferenciar dos enfoques. En las estructuras de los sindicatos, particularmente en las «secretarías de la mujer» o «secretarías de la igualdad» tiende a predominar una concepción amplia: la conciliación debería afectar a hombres y mujeres y, por tanto, no centrarse en un apartado específico del convenio, sino ser una visión transversal a lo largo de todo el entramado de las relaciones laborales 5 . También debería, dicen, entenderse como conciliación de la vida laboral y personal, como algo más amplio que lo familiar ${ }^{6}$, acepción polémica, porque se referiría a conciliación poco compatible con un sistema de producción que implique cooperación y división social del trabajo, puesto que los deseos personales difícilmente se compaginan siempre con exigencias empresariales. Con otras palabras, esto nos llevaría mucho más allá de la negociación dentro de nuestro sistema socioeconómico.

Por el contrario, los sindicalistas de empresa, en los comités, tienden a actuar, y por tanto a pensar, como si las medidas de conciliación afectaran principalmente a las mujeres porque éstas tienen obligaciones familiares que cumplir - léase, cuidado de hijos menores (lo que contempla la Ley de conciliación) y de personas mayores- y esto aparece en las entrevistas en formas sutiles, aunque hayan declarado previamente que la conciliación debe afectar a los trabajadores de ambos sexos ${ }^{7}$. Esta posición presupone mantener dichas tareas familiares como obligaciones estrictamente de las mujeres y, por tanto,

5. Véase la opinión de un miembro de un comité de empresa: «La conciliación laboral implica que el trabajo no moleste tu vida».

6. Una sindicalista de una secretaría de la mujer definía su concepción de la conciliación en los siguientes términos: «La conciliación debe ser la armonización de la vida laboral y personal, y no únicamente de la familiar, ya que las personas tienen derecho a poder realizar otras cosas además de trabajar en su empleo y de cuidar de las personas que viven con ellas, sea estudiar, hacer deporte, u otras».

7. Véase, en las entrevistas, «Es útil para facilitar el acceso de la mujer al trabajo», por ejemplo, o «de lo contrario, la solución está en las abuelas». 
asumir que seguirán en posición subordinada en el mercado de trabajo y en la profesión, al menos por dos motivos. El primero porque siempre estarán pendientes de que las empresas quieran conceder ese derecho y quizá lo hagan con restricciones o en horarios poco oportunos. El segundo porque aún en la mejor de las negociaciones, si ese derecho se consigue sólo para que las mujeres puedan cumplir mejor con sus deberes familiares, éstos seguirán siendo una barrera en la igualdad de oportunidades con los hombres, por mucha conciliación que se permita. Por ejemplo, alguna empresa ha creado un "comité de igualdad de oportunidades», lo que en principio puede sonar muy avanzado, y entre las medidas que dicho comité está desarrollando aparecen algunas como «posibilitar que las mujeres no realicen turnos de noche» o que «tengan un horario que les permita quedar libres el viernes por la tarde». Dichas mujeres trabajadoras, reafirmadas dentro de la empresa como responsables de la vida familiar, podrán conciliar sus obligaciones laborales con sus obligaciones familiares, pero nunca su vida personal, ni dejarán de ser las únicas que deben asumir las obligaciones familiares, lo cual será siempre un freno en su actividad laboral y a sus deseos personales.

Esta concepción dominante en el lugar de trabajo deriva, en buena medida, de que así siguen pensando la mayoría de trabajadores y trabajadoras. «Son pocos los trabajadores hombres que se deciden a ejercer ese derecho, ni siquiera compartidamente con sus mujeres», señala un entrevistado. Pero son más las «mujeres que tienden a pensar que ése es un derecho de ellas, por haber parido» (¿y qué decir cuando lo que se requiere hacer es cuidar a los mayores?). Por supuesto que en la práctica concreta se entiende que lo que sucede es que la reducción de la jornada la toma la mujer, porque, con frecuencia, su salario es menor y ello afectará menos a la economía doméstica. Por lo cual, el derecho a la conciliación va inseparablemente unido a la igualdad de derechos y condiciones laborales entre hombres y mujeres, en particular en lo referente a los salarios. Pero la argumentación también podría ser hecha a la inversa, esto es, si la mujer, durante seis años —o más si encadena varios hijos-, convierte su jornada en parcial por la dedicación a los hijos, se le esfuman las posibilidades de formarse, promocionar y conseguir un salario más alto en la empresa. A pesar de todo, una realidad aparece con mucha fuerza en la investigación: quienes piden tiempo para conciliar su trabajo con «obligaciones familiares» siguen siendo muy mayoritariamente las mujeres.

La propia posición de las empresas frente a la conciliación puede empujar a los sindicalistas a concebirla como algo más específico de las mujeres que de los hombres. Las empresas, particularmente las pequeñas, suelen ser reacias a la aplicación de la Ley de conciliación, porque ello les obliga a hacer cambios en la organización del trabajo y en la contratación que ven problemáticos ${ }^{8}$. En particular en el sector del comercio, donde la conciliación coherentemente

8. Como afirma un sindicalista, el empresario hace el recorte para «no perjudicar al funcionamiento de la empresa». 
requeriría que los contratos de substitución se hicieran en sábados y domingos, dado que ésos son los días en que más se necesitará dedicarse al cuidado de algunos miembros de la familia. Aunque el costo de la mano de obra de substitución no sea superior, las empresas sí pueden temer que el rendimiento de nuevos trabajadoreso trabajadoras pueda ser menor. Pero cuando las empresas asumen esa demanda, también suelen tender a concebirla en términos de derecho de las mujeres. Más allá de las razones ideológicas, que son las mismas para el empresario que para el conjunto de la sociedad, puede convenir económicamente a la empresa que la conciliación la tomen las mujeres, de manera que a los hombres se les pueda seguir exigiendo dedicación sin límites a su trabajo.

Quizá hay que referirse aquí a supuestas «buenas prácticas» de algunas empresas (alimentarias y de comunicaciones) que, para la mayoría de los sindicalistas entrevistados, no son tales, puesto que, como mucho, significan que hay guardería laboral en la sede central o bien se concede el permiso en un horario adecuado, o se saca del turno nocturno o del trabajo del fin de semana a las mujeres que pidan dicha reducción ${ }^{9}$. En ningún caso, las llamadas «buenas prácticas» van más allá de la ley. Además, cabe subrayar que casi todas ellas son buenas prácticas pensadas para las mujeres ${ }^{10}$.

La conciliación como derecho de las mujeres no logra que éstas salgan de la posición subordinada, laboral y socialmente. Si, por el contrario, las medidas de conciliación afectaran a mujeres y hombres, siendo la pareja, cuando ésta existe, la que decidiera la fórmula a utilizar de entre las posibles y la comunicara a sus respectivas empresas, estaríamos hablando realmente de igualdad jurídica de oportunidades entre los sexos y de una forma adecuada de entender la conciliación. Por otro lado, si no hay igualdad en condiciones de trabajo entre hombres y mujeres (salariales y de promoción, entre otras), se está propiciando que quien está en peores condiciones, es decir la mujer casi siempre, sea quien asuma la conciliación. Obviamente, el paso a la igualdad real depende también de otras condiciones, entre las cuales que los hombres asuman la igualdad de obligaciones familiares de forma efectiva y real ${ }^{11}$.

Los sindicatos saben que la Ley de conciliación de 1999 da ese derecho a todos los trabajadores, pero creen que no está dotada de instrumentos que permitan que todos los trabajadores se acojan al mismo sin discriminaciones, principalmente porque lo condiciona a la salvaguarda del supuesto buen funcionamiento de la empresa o al interés de otros trabajadores: «siempre que no perjudique a otros trabajadores o al funcionamiento de la empresa» (Ley

9. «Eso de las políticas familiarmente responsables no me lo creo [...] no es nada más que un mecanismo propagandístico».

10. Así, según la opinión de un sindicalista, «buenas prácticas, pues a ver, todas aquéllas que permitan mejorar la condición de la mujer, de los jóvenes, y los discapacitados, y que entiendan que forman parte de una sociedad".

11. Según una sindicalista de una secretaría por la igualdad, «yo creo que viene determinado por lo que entendemos por familia, entre todos». 
39/1999 para promover la conciliación laboral y familiar de las personas trabajadoras. BOE 06-11-1999). Por tal razón, la acción sindical ha tendido a trascender la Ley buscando que la negociación misma asuma ese derecho.

\section{El paso de la legislación a la negociación como eje de la acción sindical sobre conciliación}

Cuando los sindicatos pretenden dar el paso a la negociación de la conciliación, las empresas suelen aducir que trasponer la legislación sobre conciliación a la negociación colectiva crea rigideces en la organización del trabajo que son difíciles de superar. En el fondo, lo que esto significa es que la negociación colectiva tiene una potencialidad que no suelen tener las leyes, puesto que el incumplimiento de lo acordado en convenio puede implicar problemas para las empresas, por ejemplo: aparición de conflictos, que podrían redundar en bajas de productividad o de rendimiento, algo que no implica habitualmente el incumplimiento de la norma legal. Ello es así puesto que ambas partes firmantes de un convenio o un acuerdo se dotan de instrumentos de intervención en el seguimiento de lo acordado que permiten detectar inmediatamente incumplimientos. Pero también porque es más probable que los trabajadores sean conscientes de ese derecho si se vincula a la negociación — con toda seguridad en el caso del convenio de empresa- que si se relaciona en una ley. «Los trabajadores pueden conocer el convenio - que se distribuye por escrito y se explica profusamente- más fácilmente que la ley», como se afirma en varias entrevistas $^{12}$.

Los sindicalistas entrevistados subrayan que la negociación colectiva garantiza mejor que la simple ley un doble efecto. En primer lugar, que puedan ser considerados como beneficiarios de las medidas de conciliación todos los trabajadores de la empresa, no sólo ni principalmente aquéllos que puedan convenir en un momento determinado a la dirección. Y, en segundo lugar, que sean los trabajadores organizados colectivamente quienes puedan intervenir en la planificación efectiva del disfrute de tal derecho. Podría suceder que, en un momento determinado, una parte importante de los trabajadores de un turno o de una sección o servicio fuesen acreedores a ese derecho, lo que implicaría problemas graves para la empresa. Si el modo como se organiza el derecho a la conciliación, en ese caso, está exclusivamente en manos de la empresa, pueden tener lugar no sólo discriminaciones, sino también pérdida efectiva del derecho por parte de algunos trabajadores. Pero si la conciliación obedece a acuerdos, se puede organizar más adecuadamente, por ejemplo, a través de una comisión ad hoc. Con la mera ley, la máxima desprotección la tendrían los trabajadores de las pequeñas empresas, las cuales fácilmente pueden argüir, con la

12. Una sindicalista explica que «a partir de la aprobación de la Ley de conciliación de 1999, sindicalmente ha existido la táctica de conseguir que, en los convenios colectivos, se reproduzca el contenido de la ley, porque el trabajador o la trabajadora se fija en los derechos que tiene a partir del convenio colectivo, mientras que las leyes no las suele conocer». 
ley en la mano, que conceder el disfrute del derecho implica «dificultad de funcionamiento para la empresa».

Pero hoy día la conciliación aún no está consolidada en la negociación de forma prioritaria, como hemos podido verificar en esta investigación y recorriendo el panorama general de la negociación de convenios. Con frecuencia, el derecho individual a la conciliación no aparece en los convenios en forma suficientemente vinculante o exigible por cada trabajador o trabajadora. Ello puede revestir varias formas. En algunos casos, los convenios se limitan a transcribir lo que dice la ley española, sin otra concreción ni adaptación que tuviera en cuenta las características de la organización del trabajo o de la plantilla, lo que convierte lo acordado en el convenio en un principio general de aplicación problemática o de difícil seguimiento. Otras veces aparece en algún tipo de programa al margen del convenio, que fácilmente cae bajo la responsabilidad de la sección de Recursos Humanos, lo que da a la empresa bastante discrecionalidad sobre su aplicación en unos u otros departamentos. En alguno de los casos estudiados es así, señalando la empresa que esa iniciativa cuenta con el apoyo del comité de empresa, por más que miembros de ésta indiquen que les gustaría que eso fuera objeto de negociación en próximos convenios, no de un programa de Recursos Humanos, lo que pone de manifiesto el margen de discrecionalidad que tal programa puede dar. La tercera fórmula es que el derecho a la conciliación sea objeto de negociación y se perfile en un acuerdo que prevé objetivos y formas para llevarlo a cabo. Ésta sería la fórmula adecuada para los sindicalistas, que, sin embargo, no es fácil de conseguir ni con frecuencia se ha conseguido.

En cualquier caso, los sindicalistas de empresa manifiestan preocupación porque ese derecho sea reconocido y respetado y por introducir ese tema en la negociación, de forma que sean superadas las actuales limitaciones que se refieren a horarios inadecuados o a insuficiencia de servicios públicos complementarios a la conciliación ${ }^{13}$. Pero es evidente que, junto a las cuestiones prioritarias referidas a condiciones laborales, el gran tema que les preocupa es el empleo y el riesgo de perder puestos de trabajo, mientras que la conciliación está en otra escala que puede parecer menos importante ${ }^{14}$. Ésta es hoy la realidad: que para los sindicalistas, y para muchos trabajadores, la conciliación es menos importante que otras demandas. Pero cabría pensar con fundamento que el derecho a la conciliación tiene fuertes vinculaciones con las condiciones laborales y con el empleo.

Lo que nos demuestra esta investigación es que el derecho a la conciliación que señala la ley puede estar recogido en convenios, pero que ello dista de ser una verdadera opción de conciliación. En efecto, la mayoría de los sindicalis-

13. Véase «Atacamos a por todas. Servicios, no discriminación, jornada, salarios, esto en la confrontación directa de la negociación colectiva».

14. Véase, «En la negociación colectiva, en primer lugar, los puntos prioritarios a conseguir son la conservación, mantenimiento y ampliación del empleo. Las cuestiones referentes a la conciliación quedarían en un segundo nivel de prioridad, inferior al primero». 
tas entrevistados admiten que hoy la ley, esté o no recogida en los convenios, se puede conseguir que se cumpla, pero con dos grandes limitaciones. La primera es la de los horarios. Una persona que trabaja a turnos necesita la posibilidad de conciliar su trabajo con unos horarios de cuidado familiar que son inflexibles ${ }^{15}$ : principalmente, cuidado de los niños, de los mayores y de los discapacitados (no puede cuidar en su turno laboral si éste es inadecuado). La segunda es la de los bajos salarios: si el salario de ambos miembros de la pareja es bajo (o lo es el de uno en el caso de hogares monoparentales), y mucho más pensando en el nivel de endeudamiento que tienen los matrimonios jóvenes, muchas personas renuncian a ejercer el derecho a la conciliación e intentan arreglarlo de otra manera, principalmente echando mano de parientes para cuidar niños y ancianos. Por tanto, existe el derecho, pero no siempre la posibilidad, de ejercitarlo. Esta cuestión pone de manifiesto que una efectiva conciliación requiere empleos de calidad, en lo que concierne a la estabilidad laboral, a la remuneración salarial o a los horarios de trabajo. La proliferación de empleos precarios, en el sentido más amplio del término (Cano, 2004), o empleos con horarios desregulados, salarios bajos y pocas prestaciones sociales va en contra de la lógica y los objetivos de la conciliación. Y esto es, a nuestro entender, un elemento importante que los sindicatos deberían tener en cuenta al definir sus estrategias.

Por otro lado, el punto nuclear en el ejercicio de la conciliación está en la organización del trabajo, que sigue siendo una fortaleza infranqueable a la negociación en casi todas las empresas ${ }^{16}$. «Sin poder intervenir en la organización laboral, tenemos que olvidarnos de consolidar la conciliación del trabajo y la vida familiar", señala una sindicalista entrevistada, reflejando acertadamente esta circunstancia. En realidad, la conciliación llevada en sus justos términos significa que el trabajador no es mera fuerza de trabajo al que se paga por la labor realizada en la empresa, sino que es una persona que, como tal, tiene también una serie de deberes y necesidades externas de las que no se desprende ni siquiera trabajando ${ }^{17}$. Esto implica una revolución en el concepto de empresa y en cómo ha de organizarse la actividad en la misma.

Lo conseguido hasta ahora en la negociación se califica de «escaso, insuficiente, poco adecuado» en casi todas las entrevistas a sindicalistas. Ello es así en parte porque los sindicatos no presionan suficientemente en este terreno y la razón de esto es que no es una prioridad para muchos trabajadores y trabajadoras, y también debido a la tendencia general dominante entre los sindica-

15. En esta misma dirección, una sindicalista comenta que «Lo importante sería tener unos mejores horarios que permitieran compatibilizar mejor la jornada laboral con las obligaciones domésticas. Si hubiera más horarios intensivos, las dificultades para conciliar serían menores. Si sabes que dispones de la mañana o de la tarde, las dificultades son menores».

16. La organización del trabajo sigue estando en manos de las empresas, de acuerdo con el texto de la mayoría de los convenios.

17. Según una miembro de la Secretaría de la Mujer: «Las personas tienen derecho a hacer otras cosas a parte de trabajar». 
tos a adaptarse a las prioridades y presiones de la empresa. Los sindicalistas mismos señalan que la meta debería ser: conseguir horarios adecuados a las necesidades externas en el momento de la reducción de jornada y ampliar el periodo de reducción de jornada laboral de seis a ocho años ${ }^{18}$. También se debería ampliar el permiso pagado de maternidad (paternidad) a veinticuatro meses, así como ubicar la hora de lactancia en la manera más adecuada a la madre. Así mismo, obtener que durante el embarazo la mujer no trabaje en turno nocturno (lo que ya facilitaría el mejor horario en el momento de la reducción, si ella ejerce el derecho), como también conseguir que la conciliación se extienda también al cuidado de los mayores y discapacitados ${ }^{19}$. Todo ello debería ser factible para hombres y mujeres, pero también para parejas de hecho. En algunas empresas, principalmente en las grandes, se comienzan a mover las aguas en esa dirección, puesto que en éstas últimas el comité de empresa no sólo tiene más capacidad de presión, sino que puede «intercambiar» ese derecho por otras prestaciones.

Desde nuestro punto de vista, hay otro aspecto al que convendría prestar atención. En la negociación de las condiciones de la conciliación, se tiende a poner casi todo el peso sobre el ámbito de la vida familiar y casi nada sobre el ámbito laboral. Es decir, se quiere alargar el periodo de reducción de jornada o bien el permiso de maternidad, etc. y se olvida que el alejamiento del trabajo durante una parte de la jornada por un largo periodo puede afectar a la promoción o a la formación. Por tal razón, podría ser lógico negociar «contraprestaciones» que garantizasen que durante ese periodo las personas que tienen reducción horaria acceden en igualdad de condiciones que el resto a cursillos, concursos, promociones, etc. Podría pensarse que la razón de que esto no sea así radica en que prevalece aún la idea de que la mujer que trabaja compatibiliza sus tareas familiares con el horario laboral que pueda, lo cual sigue siendo una visión de subordinación del trabajo de la mujer.

\section{¿Conciliación desde la gestión de los RRHH o bien desde las RRLL?}

El ejercicio concreto del derecho a la conciliación tiende a ser gestionado desde el Departamento de Recursos Humanos y, por tanto, de forma individualizada ${ }^{20}$. Este proceso se enmarca dentro de la tendencia más general hacia la individualización de las relaciones laborales, que plantea importantes problemas y retos para la acción sindical (Miguélez, 1999). En este contexto, por tanto, el ejercicio concreto del derecho a la conciliación aparece como una

18. Cabe señalar que esta demanda ha sido asumida por el Gobierno socialista en el Proyecto de Ley de igualdad de derechos entre hombres y mujeres en febrero de 2006.

19. Sólo en un $2,9 \%$ de los convenios de 2003 aparecen cláusulas sobre permisos para cuidar a personas mayores.

20. Según una sindicalista, «las relaciones laborales son de carácter fuertemente paternalista. La tradición de la empresa es llevar los temas sociales y de conciliación por la vía individual». 
cuestión entre empresa y trabajador o trabajadora, en la que el actor laboral colectivo interviene al fijar el derecho, pero no al ejercerlo. Las empresas argumentan que ha de ser así, porque es algo muy personal - «como cursos de yoga o de gestión del tiempo», señala algún sindicalista refiriéndose a la forma de pensar de ciertos mánagers_-, pero, en verdad, se trata de derechos sociales colectivos con concreción individual. De manera que si bien la oportunidad debería poder ser escogida por el trabajador o la trabajadora, la posibilidad de que se haga realidad y la forma que tome dependen de que pueda ser reivindicado colectivamente. Así como otras medidas que pueden ser individuales, porque unos las harán efectivas y otros no, como la formación, la garantía de su ejercicio proviene sólo de que pueda ser demandado colectivamente.

Una evidente consecuencia de esta gestión individual es el miedo a ejercer el derecho por parte de los trabajadores y las trabajadoras. En algunos casos, se duda si pedir la reducción por miedo a mal quistarse con la empresa, con las posibles consecuencias de esto sobre el empleo. También esa forma de gestión puede enfrentar a la trabajadora o al trabajador con sus compañeras y compañeros, dado que, en el caso de que se pida un «horario arreglado» - el único que tiene sentido, como se señalaba anteriormente-, Recursos Humanos puede hacerlo «a costa» de los horarios de otros y contribuir así a aumentar las dinámicas de segmentación y deshomogeneización de las condiciones de trabajo dentro de la empresa. Sólo hay posibilidad de resolver esos problemas si el comité puede negociar con la empresa las formas concretas en las que se tomarán los permisos y las licencias de la conciliación, lo que implica meterse en la organización del trabajo de la empresa. Aquí radica el verdadero reto de la conciliación.

\section{Concertar el contexto social, la conciliación}

Sin servicios públicos adecuados, las medidas de conciliación en la empresa puede que tengan una incidencia limitada sobre la calidad de la vida familiar y personal ${ }^{21}$. En este sentido, las políticas neoliberales de privatización o de recorte de los servicios públicos son antagónicas a los objetivos de conciliación. Entre los servicios públicos necesarios, destacan, entre otros: guarderías para los niños, posibles apoyos externos para las personas mayores y transporte adecuado cuando los turnos o la distancia convierten en demasiado alejados el hogar y el puesto de trabajo.

Hay una disputa entre sindicalistas respecto a las guarderías que también nosotros hemos podido captar. Para algunos, la guardería de empresa sería una

21. Comenta una sindicalista de una secretaría de la mujer: «Para conseguir que las personas puedan conciliar su vida laboral con el resto de su vida, es necesario, además de hacerlo en la negociación colectiva (en las permisos, licencias, etc.) y en la organización del trabajo, aunque en este aspecto es difícil legalmente, conseguir la mejora y la ampliación de distintos servicios públicos: transporte, guarderías, etc. 
solución adecuada, porque permitiría realizar una reducción menor de jornada o casi minimizarla (con la consiguiente ganancia económica), mientras que otros piensan que las guarderías de los hijos han de estar en el barrio y lo que se debe reivindicar a la sociedad es que haya más y/o a la empresa, una subvención para poder utilizarlas.

Además de servicios públicos, se requerirían políticas públicas adecuadas relativas a la familia, a la infancia y a la tercera edad. El obstáculo fundamental con el que se enfrentan muchas mujeres, y lo mismo pasaría con los hombres, que quieren reducir su dedicación laboral para conciliar sus actividades es que ello implica una reducción de salario que no pueden asumir. Si existiesen subvenciones para el cuidado de los hijos hasta una cierta edad o bien por el cuidado de los mayores, que pudiesen ser recibidas por quienes los cuidan, el obstáculo económico quedaría substancialmente rebajado, porque, en ese periodo, el salario quedaría "completado» por la subvención, sin tener que renunciar al trabajo que se podría mantener a tiempo parcial. No se trata de una utopía, puesto que en algunos países sucede algo similar. Sólo se trata de si se quiere dar a la conciliación el sentido que realmente tiene.

Todo esto nos pone de manifiesto que la conciliación ni siquiera se resuelve a través de la mera negociación colectiva, al menos tal como actualmente está planteada. Se requiere introducir medidas de concertación entre actores sociales y Administración que amplíen los periodos de permiso pagado, por los cuales las empresas pequeñas puedan tener compensaciones cuando se encuentren en tales circunstancias. Pero también que posibiliten la puesta a punto de servicios adecuados, como guarderías o servicios para gente mayor, así como mejores transportes nocturnos y otras. Se requiere un salto de calidad en el Estado de bienestar por el que la satisfacción de ciertas «necesidades nuevas» sea asumida como un derecho universal y en vistas a lo cual ya no hay la simple contrapartida del empleo, sino un empleo que permita adecuarse a dichas necesidades, un empleo de calidad.

\section{A modo de conclusión}

Los sindicalistas no son optimistas respecto a una adecuada implantación del derecho a la conciliación para hombres y mujeres, en un tiempo razonable, si seguimos con el mismo camino trazado hasta ahora. Para llegar a una formulación real de este derecho, se requeriría actuar en una triple dirección. En primer lugar, sería necesario poder intervenir en la organización del trabajo. Si los trabajadores, a través de sus representantes, no tienen la posibilidad de codecisión en la configuración de los horarios, de los turnos, de la estructura de las jornadas, de la carga de trabajo, o bien de la movilidad interna, entre otras, es muy difícil que se pueda articular el tiempo de trabajo, tanto de dentro como de fuera de la empresa. Y es que los trabajadores, antes que como recursos, deberían de ser considerados como personas (Riconà y Pontí, 2004). Pero, interviniendo en la organización del trabajo, las cosas no se arreglan si, al mismo tiempo, no hay guarderías, buenos transportes, apoyos para las tareas de 
proximidad, es decir, si no hay unas políticas y unos servicios que posibiliten conciliar ambos trabajos sin tener que renunciar al primero. Dicho con otras palabras, si no hay un avance substancial en el Estado de bienestar y no se pone fin a las políticas de recorte y reducción de los servicios públicos. En tercer lugar, se requieren avances en el terreno ideológico y en la consiguiente práctica, en particular, en la implicación de los hombres en los trabajos domésticos y del cuidado familiar. Mientras éstos no lo hagan, tanto los sindicatos como las empresas entenderán la conciliación como «algo que facilita a las mujeres el ejercicio de sus dos trabajos».

Habría que agregar algo más. Los tiempos de la ciudad ya no son los tiempos de la industria, sino que están cambiando aceleradamente (Torns y Miguélez, 2002). Son tiempos de actividad productiva mucho más flexibles, más invasivos de los otros tiempos de la vida, menos estándar. Frente a ello, se requiere volver a rediscutir la política de los tiempos y probablemente no se pueda llegar a soluciones satisfactorias para mucha gente si no se entra en un retoque a fondo de la organización del trabajo y en preguntarse quién tiene facultades para tomar decisiones sobre dicha organización. Es decir, si no se avanza seriamente en la democracia económica.

Las mujeres que pueden conciliar en algún grado son las que ganan buenos sueldos, ellas y sus parejas, y que contratan a alguien para el «trabajo necesario» - en muchos casos, mujeres inmigrantes_, es decir, las que ya conciliaban por su cuenta.

Parece que si queremos conciliar vida laboral y no laboral deberíamos estudiar otras posibilidades y desarrollar otras políticas. Por un lado, es importante plantear la conciliación para todos, hombres y mujeres, individualizadamente pero entendiéndola como un derecho social colectivo. Por otro, referirla a los tres tiempos de la vida cotidiana (trabajo productivo familiar y tiempo de ocio), y no sólo a los dos primeros, en los que hombres y mujeres tienen derechos y obligaciones. Las políticas deberían facilitar el reparto del «trabajo necesario» y posibilitar la igualdad de oportunidades en el trabajo remunerado, limitando la flexibilidad por el derecho de los trabajadores y las trabajadoras a los otros tiempos personales y familiares. Los sindicatos deberían plantear los tres puntos mencionados de forma simultánea, puesto que el centro de este reparto social equilibrado del tiempo sigue siendo el horario laboral.

Desde este punto de vista, parece necesario que los sindicatos adopten una estrategia más proactiva y menos adaptativa que, por un lado, busque no subordinarse y acomodarse a las necesidades y a las presiones empresariales de mayor flexibilidad y desregulación de las condiciones laborales y, por otro lado, busque defender los intereses del conjunto de una clase trabajadora entendida de forma plural y amplia, y atienda por igual a las necesidades de hombres y mujeres y a las de los colectivos de trabajadores y trabajadoras más débiles y con menor poder de negociación. 


\section{Bibliografía}

CANO, E. (2004). «Formas, percepciones y consecuencias de la precariedad». Mientras Tanto, n. ${ }^{\circ}$ 93, invierno, p. 67-82.

CES (2004). «Résolution de la CES en vue du Sommet du printemps 2004 de l'UE». Résolution du Comité Exécutif, 17 y 19 de abril.

CES (2004). «Parvenir à une amélioration quantitative et qualitative de l'emploi en plaçant l'Europe sociale au coeur de la stratégie de Lisbonne». Résolution du Comité Exécutif, 1 de diciembre.

COMMISSION EUROPÉENNE (2002). L'emploi en Europe. Bruselas.

DiCKENS, L. (2000). «Collective bargaining and the promotion of gender equality at work: opportunities and challenges for trade unions». Transfer, 2.

ETXEZARRETA. M. (2001). Una panorámica crítica de la UE: un texto de divulgación. Inédito.

GoBIn, C. (1997). L’Europe Sindícale. Bruselas: Éditions Labor.

GRUPO QUIT (2004). L'organització social del temps. Seminari Departament de Sociologia. Universitat Autònoma de Barcelona, 30 de enero.

Heinen, J. (2004). «L'Union Européenne face aux inégalités de genre». Contre Temps, n. ${ }^{\circ}$ 9, febrero, p. 158-168.

Hubert, A. (1998). L'Europe et les femmes-Identités en mouvement. Rennes: Apogée. Hyman, R.; Ferner, A. (eds.) (1994). New frontiers in European industrial relations. Oxford Cambridge: Blackwell.

LozARES, C.; MiguÉLEZ, F. (2004). «Time work flexibility against time of life inflexibility». International Symposium on Working Time. París, febrero.

MarTín, A.; Ross, G. (2001). "Trade Union Organizing at the European Level: The Dilema of Borrowed Resources». En: IMIG, D.; TARRow, S. (eds.). Contentious Europeans. Oxford: Rowman and Littlefield.

Miguélez, F. (1999). «Los sindicatos». En: MiguéLEZ, F.; PRieto, C. (eds.). Relaciones de empleo en España. Madrid: Siglo XXI.

MiguéLEZ, F. (1999). «Presente y futuro de la acción sindical en España». En: MiguéLEZ, F.; PRIETO, C. (eds.). Relaciones de empleo en España. Madrid: Siglo XXI.

MiguéLEZ, F. (2004). «La flexibilidad laboral». Trabajo, n. ${ }^{0} 13$, junio.

MiguéLEZ, F. (2004a). «Los veinte últimos años de las relaciones laborales». Mientras Tanto, n. ${ }^{\circ}$ 93, invierno, p. 45-56.

Ricona, C.; PONTí, F. (2004). No somos recursos, somos humanos. Barcelona: Granica.

RigBY, M. (2004). «Equilibrio entre trabajo y vida no laboral». Trabajo, n. ${ }^{\circ} 13$.

Rossi, I.; DEMETRIADES, S. (2002). «Reconciliation of work and family life and collective bargainning». En: <www.eiro.eurofound.eu.int>.

RUberY, J. (1997). «What Do Women want from Full Employment?». En: PHILPOTT, J. (eds.). Working for full employment. Londres: Routledge.

SCHulten, T. (2000). «New Provisions on Parental Leave and Childcare Payments». En: <www.eiro.eurofund.ie>.

Tiernan, S. (2004). «ETUC Policies on Motherhood and Work». Transfer, n. 10.

Torns, T.; Miguélez, F. (eds.) (2001). Temps i Ciutat. Barcelona: Consell Econòmic i Social de Barcelona.

TORNS, T. (2006). «Las políticas del tiempo: un reto para las políticas del Estado de bienestar». Trabajo, n. ${ }^{\circ} 13$.

Trentini, M. (2000). «New Law Adopted on Parental Leave». En: <www.eiro.euro fund.ie>. 